\title{
Distributed Adaptive Virtual Impedance for Reactive Power Sharing in Microgrid
}

\author{
Harini $\mathrm{M}^{1} \mid$ Dr.S.Chitra ${ }^{2}$
}

${ }^{1}$ PG Scholar, Department of EEE, Government College of Technology, Coimbatore, Tamil Nadu, India.

${ }^{2}$ Assistant Professor,Department of EEE, Government College of Technology, Coimbatore, Tamil Nadu, India.

\section{To Cite this Article}

Harini M and Dr.S.Chitra, "Distributed Adaptive Virtual Impedance for Reactive Power Sharing in Microgrid", International Journal for Modern Trends in Science and Technology, 6(8): 135-140, 2020.

\section{Article Info}

Received on 02-July-2020, Revised on 25-July-2020, Accepted on 02-August-2020, Published on 09-August-2020.

\section{ABSTRACT}

The concept of microgrid has been developed to realize flexible coordination control among Distributed Generation (DG) units, improve the power quality supplied to customers. The problem such as the power quality and the system stability due to the intermittency of the renewable energy sources and the fluctuating load profile. The reactive power sharing done by droop control method but reactive power is not accurately shared if there is a local load at each DG. In this paper adaptive virtual impedance control is used to improve the power control stability and sharing performance of real and reactive power sharing is compared by using MATLAB/Simulink environment. Simulation results shows the effectiveness of the proposed method is achieving load reactive power sharing and the voltage restoration is settles in less time.

KEYWORDS: Switched reluctance motor, SRM control, Speed control, ZETA Converter

\section{INTRODUCTION}

With the growing concerns about the renewable energy sources have been integrated in microgrid. However, the high penetration and increasing capacities of the renewable energy sources exhibit many challenging problems, such as the power quality, system stability and fluctuating load profile [1] and[4]. To cope with these problems, the microgrid concept has been developed to realize flexible coordination control among DG units. Microgrid that improve the availability and the power quality supplied to customers could be operated in grid-connected mode and islanded mode. In the case of islanded mode, the load demand should be properly shared by DG units. To satisfy the power sharing requirement without critical communications, the droop control method which mimics the behavior of a synchronous generator in microgrids has been widely adopted. The droop control method that does not rely on external communication links enables "plug-and-play" of DG units [3] and [2], [5]. Recently, the hybrid microgrid architecture has been proposed to realize the load power sharing among different phases. However, in practical situations, although the frequency droop control can realize the accurate active power sharing, the voltage droop control typically results in poor reactive power sharing due to the mismatched line impedances and the difference of DG inverter parameters[2], [3]. To improve the reactive power sharing accuracy in islanded microgrids, various control methods have been developed. The approach based on signal injection technique was proposed in and to achieve the accurate reactive power sharing [6]. However, it is difficult to be applied to the microgrid that contains more than two DG units due to the complication of implementation in generating and processing of the signal. In the reactive power sharing accuracy was improved by regulating the droop coefficient. But the load slope estimation during the 
grid-connected mode is used to regulate the droop coefficient in islanded mode [7], [8]. The concept of virtual impedance was introduced to improve the power control stability and the power sharing accuracy. These method eliminated the mismatch of the line impendence. Therefore, the virtual impedance control based on the estimation of the line impedance was proposed in and several other control method which employ adaptive virtual impedance were developed [16],[20],[7]. In each DG adaptively tunes the virtual impedance through the integrator by estimating the effect of the voltage drop mismatch. In, the virtual impedance was determined according to the transient active power variation introduced by injecting frequency disturbances.

In addition, the DG output voltage deviation is produced due to droop control and the added virtual impedance, and so the output voltage of each DG is not regulated at the rated voltage across microgrid if the influence of the line impedance is taken into account [20] [12] [15]. Although these works provide the basis for conventional droop control, the reactive power sharing with distributed virtual impedance regulation and the average voltage restoration are not proposed with consideration of the line impedance.

In this paper, adaptive virtual impedance control strategy is proposed for low voltage microgrid by using conventional droop control method [10] [13]. The virtual impedance to compensate the mismatched line impedance for accurate reactive power sharing. The proposed method objective is focus on developing power sharing control techniques for microgrids with multiple distributed generation units. The power sharing within these generation units will be studied when the microgrid is operating in island mode. In order to achieve load reactive power and voltage restoration and eliminate the use of adaptive virtual impedance approach .The performance will be assessed through simulations [18], [20].

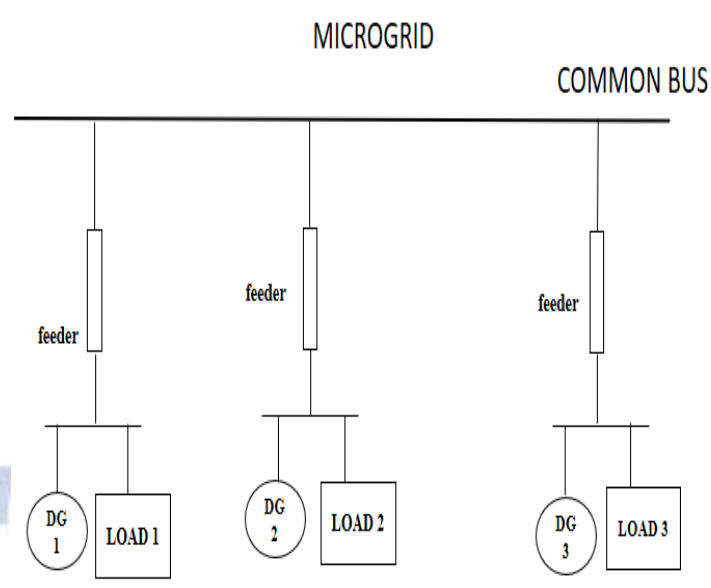

Fig.1. Test system

\section{POWER FLOW CONTROL CIRCULATING CURRENT}

The use of paralleled inverters is advantage when it comes to increased reliability and power rating, and is also one of the characteristics of islanded microgrids. However, this organization of DG units also brings challenges. The generation of circulating currents is one of these, which will be presented in this section. DG units operating in parallel with different output voltages, output impedance or phase can cause current not only flowing from the generation to the loads but also between the generation units [14] [15]. In a traditional power grid with large synchronous generators, the line smaller line impedance in microgrid make the circulating current a major challenges. The circulating current can lead to increased losses and overloaded inverters, in addition to reduction in power quality and efficiency.

In unbalanced current can occur between the phases making the challenge even more complex[10]. However not considered in this paper due to the assumption of balanced three- phases system. The assumption also makes the definition of circulating current presented in valid for a three-phase system, which will be used further in the analyses.

\section{DROOP CONTROL}

Droop control algorithms seem promising in order to accommodate the aforementioned objectives for the microgrid. Therefore chosen to be used in the primary control loop. The frequency and voltage levels communicate if there is under or over production in the microgrid, which is used to adjust the power supplied by the inverters .this is done through a high level of control, by adjusting 
the set points for active and reactive power [11] [13]. The droop control is located at the inverters, where the operation of each unit will adapt to the entire system. Because of this, the control strategy is well suited for expansions in the grid. Since the utilization of droop control does not require high-speed communication, this may lead to significant reductions in costs compare to a centralized controller in addition to high reliability[11] [13]. The concept of droop control, a power flow through the line . with an output impedance represented by $Z$, the active and reactive power flow in the equation given by

$$
\begin{aligned}
& P=\frac{v 1^{2}}{z} \cos \theta-\frac{v 1 v 2}{z} \cos (\theta+\delta) \\
& Q=\frac{v 1^{2}}{z} \sin \theta-\frac{v 1 v 2}{z} \sin (\theta+\delta)
\end{aligned}
$$

Where, $v 1$ and v2 are the voltage of bus, $\partial$ is the power angle $Q$ is the angle between output impedance and $z$ is impedance.

\section{CONVENTIONAL DROOP CONTROL}

Even if most low-voltage microgrids have line impedances dominated by a resistive term, components such as transformer and filters can contribute to a more inductive behavior. In case of small line impedance, the output impedance can become predominantly inductive. With inductive output impedances, the conventional droop control method can be used to control power sharing. The output impedance is purely inductive. This makes the impedances $Z$, equal to pure reactance, $X$. The impedance angle will be equal to 90 . The power angle is assumed to be small. The power angle which is directly related to the frequency is dependent on the active power. The voltage droop, $\mathrm{V} 1-\mathrm{V} 2$ is related to the reactive power. This is the basis for the conventional droop characteristics, as represented in equation (3) and (4).

$$
\begin{aligned}
& \omega_{i}=\omega_{0}-m_{i} P_{i} \\
& E_{i}=E_{0}-n_{i} Q_{i}
\end{aligned}
$$

Where, $E_{\mathrm{i}}$ is the rated voltage, $\omega_{\mathrm{I}}$ is the rated frequency, $m_{i}$ is the droop frequency, $n_{i}$ is the amplitude coefficient, $P_{i}$ and $Q_{i}$ is the active and reactive power .

The characteristics is based on a rated voltage and frequency, denoted $\mathrm{E}_{\text {ref }}$ and $\mathrm{I}_{\text {ref. }}$. The active and reactive droop coefficients are given as $m_{i}$ and $n_{i}$ [9] [15]. They decide the relation between frequency and active power, voltage and reactive power, respectively. $P_{i}$ and $Q_{i}$ are the set points for active and reactive power. These are determined by a higher level of control, which normally is dependent on communication in order to adapt to the load of the system. with close to purely inductive output impedances, the conventional droop control method is effective way to adjust frequency and voltage relative to the supplied power. [17] [18]
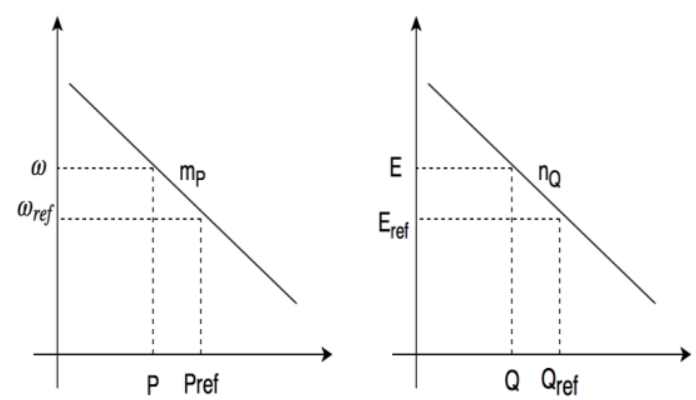

\section{Fig. 2. Characteristic of the conventional droop control}

However, the conventional droop control method is not always true since the line impedance is predominantly resistive for the low-voltage microgrid where the droop control may cause the coupling between the active and reactive power. In order to present mainly inductive impedance even in the low-voltage microgrid, the virtual inductive impedance is introduced into primary control loop the DG to realize the decoupled active and reactive power flows.

\section{VIRTUAL IMPEDANCE}

Virtual impedance is a control mechanism which is frequency used for this purpose, often in combination [17][19] with droop control. In additional to power sharing, virtual impedance are used converter control for applications such as harmonic voltage compensation and increased stability. The concept is divided into two categories in: inner and outer virtual impedance.

Inner virtual impedance-loops are used for harmonic compensations and the signal from the loop is applied at the PWM. Harmonic voltage compensation, control of power flow, mitigation of unbalanced voltage and sharing between nonlinear load in power system are done by the use of outer virtual impedance, which modifies the reference signal the controller. Because of its many applications, the value of this impedance has to be decided based on its purpose the value of the virtual impedance are studied in order to optimize the microgrids performance. The virtual impedance can help to keep the voltage within certain limits, 
have sufficient reactive power sharing provide system damping[20] [17] [15]. and decouple the active and reactive power. And its behavior of an actual impedance, without experiencing losses. The utilization of outer virtual impedance loop is studied in order to improve power sharing between linear loads. First an elaboration on outer virtual impedance techniques will be performed. In the following, outer virtual impedance is simply denoted virtual impedance. This approach can both change the magnitude and the impedance angle of the equivalent output impedance. The selection of the virtual impedance have to be done carefully, both when it comes to the relation between the resistive and reactive components, but also regarding the impedances total size[11] [12] and [16].

A distinct disadvantage of applying virtual impedances is their tendency of reducing the voltage reference. This has to be taken into consideration when the value of the impedance is selected. A thorough review of advantages and disadvantage regarding virtual impedance in combination with droop control is given by

$$
\Delta V r e f=V *-\Delta V v=V *-Z v . I \text { output }
$$

Where $\mathrm{V}^{*}$ is denoted the voltage reference from the droop controller, $\Delta \mathrm{V}_{\mathrm{v}}$ is the voltage droop due to the virtual impedance, $I_{\text {output }}$ is the output current, $Z_{\mathrm{v}}$ is the virtual impedance and $\mathrm{V}_{\text {ref }}$ is thee voltage reference applied to the inner control.

\section{SIMULATION AND RESULT}

\section{SIMULATION OF CONTROLLER}

This chapter discus about the simulation, output and result figure 3.1 , the simulation of controller, the $\mathrm{DC}$ voltage converted into $\mathrm{AC}$ voltage by using inverter circuit IGBT.

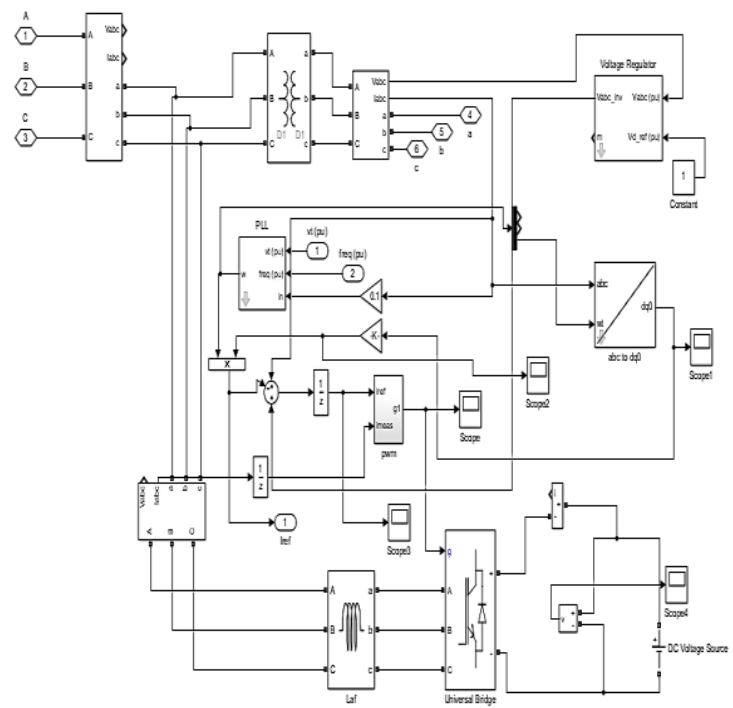

Figure 3. simulation of controller

The three phase AC voltage is given to the load by using transformer, output power is controlled by controller design in Fig 3. The current and voltage value is taken as references, adaptive virtual impedance is injected to controller that causes the droop in the voltage, the active and reactive power of load is control by virtual impedance control.

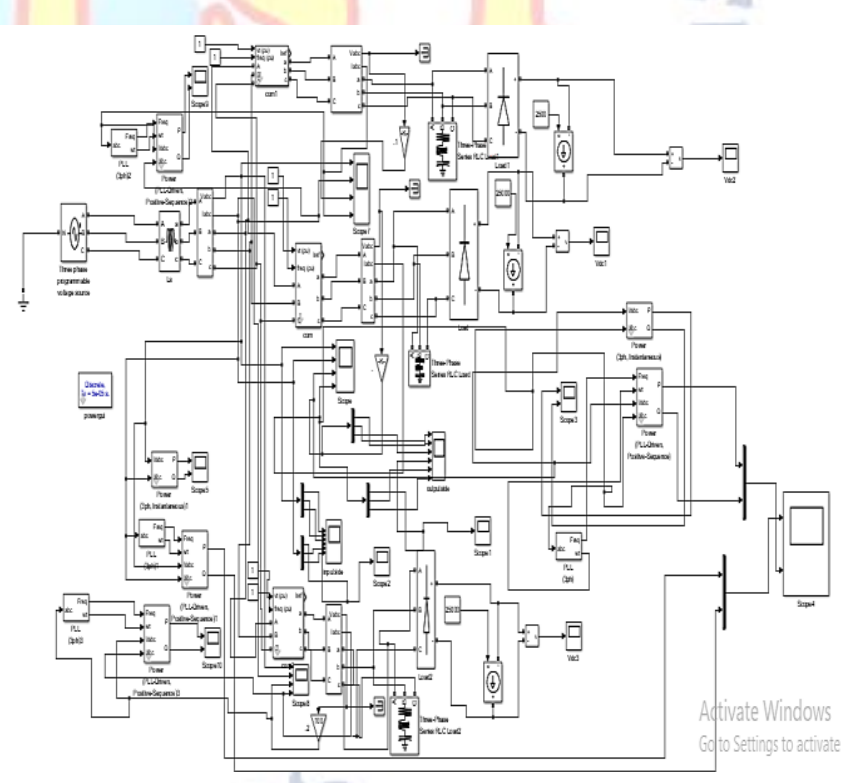

Fig 4. Detailed configuration of a DG unit using the proposed control method.

The output of the regulator and abc and dqo transformation given to PWM, generated pluses is given to inverter. Simulation of adaptive virtual impedance control for reactive power sharing DC source and loads are connected to grid the generating power is given to the load the reactive power is injected, compensation and controlled by 
the controller reactive power is settles fast compare to before injection Fig 5 .
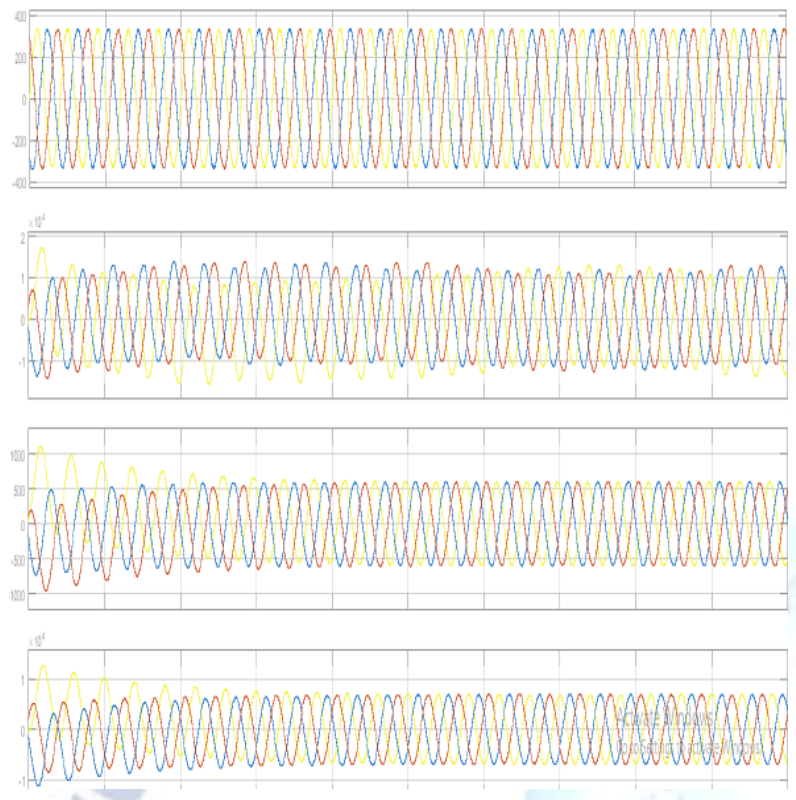

Fig 5. Waveforms of voltage and current

The output of three voltage and current of DG, with time after injection the power reduce in voltage and current settled uniformly Fig 6.

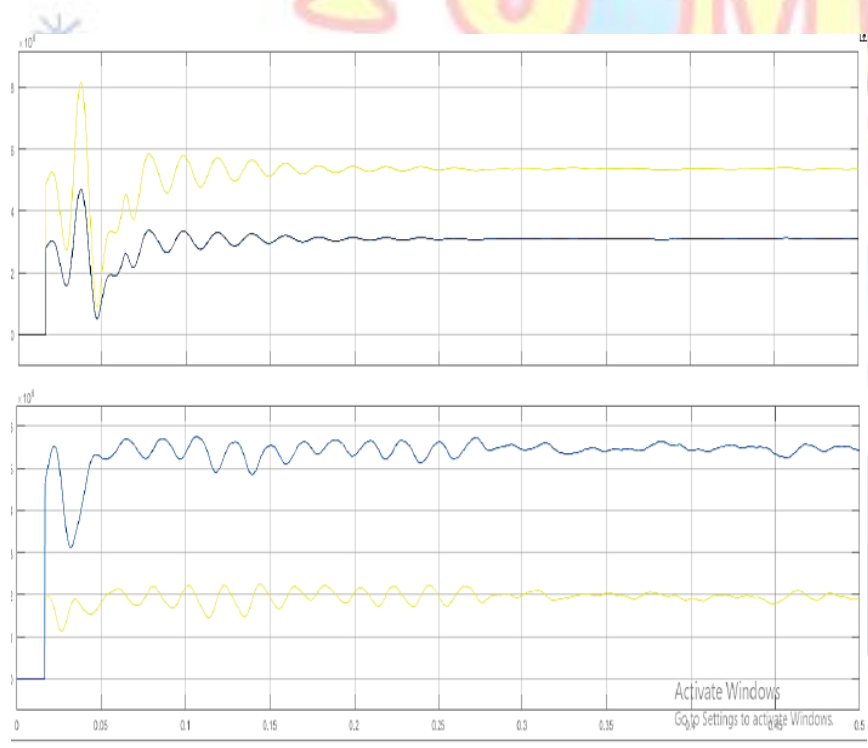

ractive power -

Fig 6. Waveforms of active and reactive power

Voltage and time, after compensating reactive power is settled less in time sec when compare to before compensation power, the settle time of reactive power is $0.2 \mathrm{sec}$.
Table 1. System parameter

\begin{tabular}{|c|c|}
\hline Parameter & Value \\
\hline $\begin{array}{l}\text { Grid voltage } \\
\text { and frequency }\end{array}$ & $450 \mathrm{~V}, 50 \mathrm{HZ}$ \\
\hline Regulator gain & $\begin{array}{l}K_{p}=180 \\
K_{i}=3200 \\
K_{d}=1\end{array}$ \\
\hline Loads & $\begin{array}{l}C=100 \\
R=10^{*} 10^{6}\end{array}$ \\
\hline DG & $450 \mathrm{~V}$ \\
\hline
\end{tabular}

Table 2. Reactive power after compensation

\begin{tabular}{|l|l|}
\hline Time $(\mathrm{s})$ & Voltage $(\mathrm{v})$ \\
\hline 0.05 & $8^{*} 10^{6}$ \\
\hline 0.1 & $5.6^{*} 10^{6}$ \\
\hline 0.2 & $5.4^{*} 10^{6}$ \\
\hline 0.3 & $5^{*} 10^{6}$ \\
\hline 0.4 & $5^{*} 10^{6}$ \\
\hline 0.5 & $5^{*} 10^{6}$ \\
\hline
\end{tabular}

\section{CONLUSION}

An islanded microgrid system is modelled in order to investigate power sharing between paralleled DG units with different output impedances. In the studied system, three DG units connected through inverters are modelled to feed one linear load. This thesis shows the distributed control structure, including the inner- and primary control. The inner control includes voltage and current control, while the primary control contains a droop control algorithm which takes care of voltage and frequency levels. In this loop, the output impedances of the inverters are modified by use of virtual impedances, which contribute to improved power sharing among the DG. To realize proportional accurate reactive power sharing among DGs, the virtual impedance has been adaptively regulated to eliminate the reactive power sharing mismatch. The distributed adaptive virtual impedances keep the equal droop output voltages of DGs to suppress effectively the circuiting currents among DGs while improving the accuracy of the reactive power sharing in spite of the mismatched feeder impedances. This control method that requires a sparse communication structure which is less sensitive to the failure of communication links can make the microgrid more reliable. Simulation results have been given to verify the active and reactive power sharing strategy. The performance of the real and reactive power sharing with adaptive virtual impedance 
controller is compared with the performance of the real and reactive power sharing conventional approach by using MATLAB / Simulink environment. The reactive voltage for proposed system settles in less time when compared to the conventional approach.

\section{FUTURE SCOPE}

The adaptive virtual impedance becomes complex when the actual impedance is not given. The disadvantage in this method is analysed and can be improved for the future project.

\section{REFERENCES}

[1] H. Zhang, J. Zhang, G.-H. Yang, and Y. Luo, "Leader-based optimal coordination control for the consensus problem of multi agent differential games via fuzzy adaptive dynamic programming," IEEE Trans. FuzzySyst., vol. 23, no. 1, pp. 152-163, Feb. 2015.

[2] R.Olfati-Saber and R.M.Murray, "Consensus problems in networks of agents with switching topology and time-delays," IEEE Trans. Autom.Control, vol. 49, no. 9, pp. 1520-1533, Sep. 2004

[3] A. Bidram, A. Davoudi, F. L. Lewis, and Z.Qu, "Secondary control of microgrids based on distributed cooperative control of multi-agent systems," IET Gener TransmDistrib., vol. 7, no. 8, pp. 822-831, Aug. 2013.

[4] A. Bidram, A. Davoudi, F. L. Lewis, and J.M. Guerrero, "Distributed cooperative secondary control of microgrids using feedback linearization,"IEEE Trans. Power Syst., vol. 28, no. 3, pp. 3462-3470,Aug. 2013

[5] Y. Xu and W. Liu, "Novel multi agent based load restoration algorithm for microgrids," IEEE Trans. Smart Grid, vol. 2, no. 1, pp. 152-161, Mar. 2011.

[6] Y. Xu, W. Liu, and J. Gong, "Stable multi-agent-based load shedding algorithm for power systems," IEEE Trans. Power Syst., vol. 26, no. 4, pp. 2006-2014, Nov. 2011.

[7] J. W. Simpson-Porco, F. Dörfler, and F. Bullo, "Synchronization and power sharing for droop-controlled inverters in islanded microgrids," Automatica, vol. 49, no. 9, pp. 2603-2611, Sep. 2013.

[8] F. L. Lewis and V. L. Syrmos, Optimal Control. Hoboken, NJ, USA: Wiley, 1995.

[9] Z. Qu, Cooperative Control of Dynamical Systems: Applications to Autonomous Vehicles. New York, NY, USA: Springer, 2009.

[10] H. Han, Y. Liu, Y. Sun, M. Su, and J. M. Guerrero, "An improved droop control strategy for reactive power sharing in islanded microgrid," IEEETrans. Power Electron., vol. 30, no. 6, pp. 3133-3141, Jun. 2015.

[11] D. P. Spanos, R. Olfati-Saber, and R. M. Murray, "Dynamic consensus for mobile networks," in Proc. 16th Int. Conf. Fed. Autom. Control, Prague, Czech Republic, 2005, pp. $1-6$.

[12] J. M. Guerrero, J. C. Vasquez, J. Matas, L. G. de Vicuña, and M. Castilla, "Hierarchical control of droop-controlled AC and DC microgrids-A general approach toward standardization," IEEE Trans.Ind. Electron., vol. 58, no. 1, pp. 158-172, Jan. 2011.

[13] A. Bidram and A. Davoudi, "Hierarchical structure of microgrids control system," IEEE Trans. Smart Grid, vol. 3, no. 4, pp. 1963-1976, Dec. 2012.
[14] K. De Brabandere et al., "A voltage and frequency droop control method for parallel inverters," IEEE Trans. Power Electron., vol. 22, no. 4, pp. 1107-1115, Jul. 2007.

[15] N. Pogaku, M. Prodanovic, and T. C. Green, "Modeling, analysis and testing of autonomous operation of an inverter-based microgrid," IEEETrans. Power Electron., vol. 22, no. 2, pp. 613-625, Mar. 2007.

[16] C. K. Sao and P. W. Lehn, "Autonomous load sharing of voltage source converters," IEEE Trans. Power Del., vol. 20, no. 2, pp. 1009-1016, Apr. 2005.

[17] J. C. Vasquez, J. M. Guerrero, M. Savaghebi, J. Eloy-Garcia, and R. Teodorescu, "Modeling, analysis, and design of stationary reference frame droop-controlled parallel three-phase voltage source inverters," IEEE Trans. Ind. Electron., vol. 60, no. 4, pp. 1271-1280, Apr. 2013.

[18] A. Mehrizi-Sani and R. Iravani, "Potential-function based control of a microgrid in islanded and grid-connected models," IEEE Trans. PowerSyst., vol. 25, no. 4, pp. 1883-1891, Nov. 2010.

[19] Q. Sun, J. Zhou, J. M. Guerrero, and H. Zhang, "Hybrid three phase/ single-phase microgrid architecture with power management capabilities," IEEE Trans. Power Electron

[20] C.-T. Lee, C.-C. Chu, and P.-T. Cheng, "A new droop control method for the autonomous operation of distributed energy resource interface converters," IEEE Trans. Power Electron., vol. 28, no. 4, pp. 1980-1993,Apr. 2013.
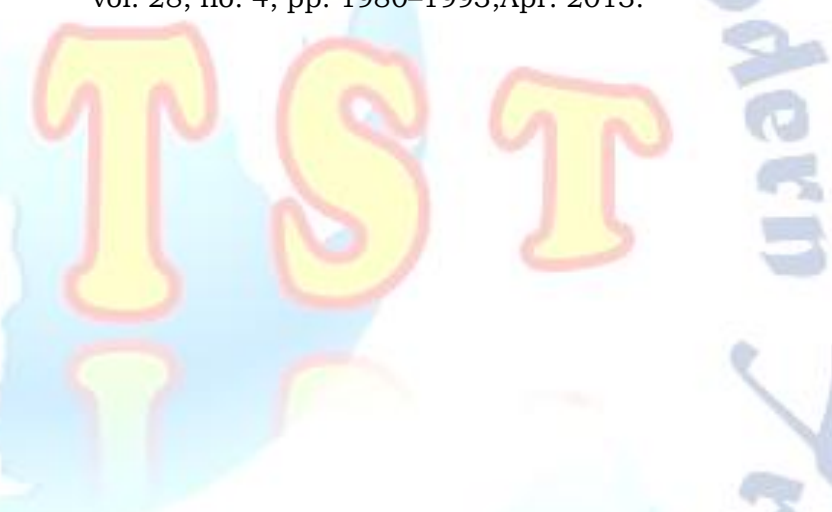